CUBO A Mathematical Journal

Vol.21, No 01, (37-48). April 2019

\title{
Commutator criteria for strong mixing II. More general and simpler
}

\author{
S. RICHARD 1 \\ Graduate school of mathematics, \\ Nagoya University, \\ Chikusa-ku, Nagoya 464-8602, Japan \\ richard@math. nagoya-u.ac.jp \\ R. TIEDRA DE Aldecos 2 \\ Facultad de Matemáticas, \\ Pontificia Universidad Católica de Chile, \\ Av. Vicuña Mackenna 4860, Santiago, Chile \\ rtiedra@mat.puc.cl
}

\begin{abstract}
We present a new criterion, based on commutator methods, for the strong mixing property of unitary representations of topological groups equipped with a proper length function. Our result generalises and unifies recent results on the strong mixing property of discrete flows $\left\{\mathrm{U}^{\mathrm{N}}\right\}_{\mathrm{N} \in \mathbb{Z}}$ and continuous flows $\left\{\mathrm{e}^{-i \mathrm{tH}}\right\}_{\mathrm{t} \in \mathbb{R}}$ induced by unitary operators $\mathrm{U}$ and self-adjoint operators $\mathrm{H}$ in a Hilbert space. As an application, we present a short alternative proof (not using convolutions) of the strong mixing property of the left regular representation of $\sigma$-compact locally compact groups.
\end{abstract}

\footnotetext{
${ }^{1}$ Supported by the grant Topological invariants through scattering theory and noncommutative geometry from Nagoya University, and by JSPS Grant-in-Aid for scientific research (C) no 18K03328, and on leave of absence from Univ. Lyon, Université Claude Bernard Lyon 1, CNRS UMR 5208, Institut Camille Jordan, 43 blvd. du 11 novembre 1918, F-69622 Villeurbanne cedex, France.

${ }^{2}$ Supported by the Chilean Fondecyt Grant 1170008.
} 


\section{RESUMEN}

Presentamos un nuevo criterio, basado en métodos de conmutadores, para la propiedad de mezcla fuerte de representaciones unitarias de grupos topológicos dotados de una función de longitud propia. Nuestro resultado generaliza y unifica resultados recientes acerca de la propiedad de mezcla fuerte de flujos discretos $\left\{\mathrm{U}^{\mathrm{N}}\right\}_{\mathrm{N} \in \mathbb{Z}}$ y flujos continuos $\left\{\mathrm{e}^{-i \mathrm{tH}}\right\}_{\mathrm{t} \in \mathbb{R}}$ inducidos por operadores unitarios $\mathrm{U}$ y operadores autoadjuntos $\mathrm{H}$ en un espacio de Hilbert. Como aplicación, presentamos una demostración corta alternativa (sin usar convoluciones) de la propiedad de mezcla fuerte de la representación regular de grupos localmente compactos $\sigma$-compactos.

Keywords and Phrases: Strong mixing, unitary representations, commutator methods.

2010 AMS Mathematics Subject Classification: 22D10, 37A25, 58J51, 81Q10. 


\section{Introduction}

In the recent paper [14], itself motivated by the previous papers [8, 12, 13, 15, it has been shown that commutator methods for unitary and self-adjoint operators can be used to establish strong mixing. The main results of [14] are the following two commutator criteria for strong mixing. First, given a unitary operator $\mathrm{U}$ in a Hilbert space $\mathcal{H}$, assume there exists an auxiliary selfadjoint operator $A$ in $\mathcal{H}$ such that the commutators $\left[A, U^{N}\right]$ exist and are bounded in some precise sense, and such that the strong limit

$$
\mathrm{D}_{1}:=\mathrm{s}_{\mathrm{N} \rightarrow \infty} \frac{1}{\mathrm{~N}}\left[A, \mathrm{U}^{\mathrm{N}}\right] \mathrm{U}^{-\mathrm{N}}
$$

exists. Then, the discrete flow $\left\{\mathrm{U}^{\mathrm{N}}\right\}_{\mathrm{N} \in \mathbb{Z}}$ is strongly mixing in $\operatorname{ker}\left(\mathrm{D}_{1}\right)^{\perp}$. Second, given a selfadjoint operator $H$ in $\mathcal{H}$, assume there exists an auxiliary self-adjoint operator $A$ in $\mathcal{H}$ such that the commutators $\left[A, \mathrm{e}^{-\mathrm{itH}}\right]$ exist and are bounded in some precise sense, and such that the strong limit

$$
\mathrm{D}_{2}:=\mathrm{s}_{\mathrm{t} \rightarrow \infty} \frac{1}{\mathrm{t}}\left[\mathrm{A}, \mathrm{e}^{-i \mathrm{tH}}\right] \mathrm{e}^{i \mathrm{tH}}
$$

exists. Then, the continuous flow $\left\{\mathrm{e}^{-i \mathrm{tH}}\right\}_{\mathrm{t} \in \mathbb{R}}$ is strongly mixing in $\operatorname{ker}\left(\mathrm{D}_{2}\right)^{\perp}$. These criteria were then applied to skew products of compact Lie groups, Furstenberg-type transformations, time changes of horocycle flows and adjacency operators on graphs.

The purpose of this note is to unify these two commutator criteria into a single, more general, commutator criterion for strong mixing of unitary representations of topological groups, and also to remove an unnecessary invariance assumption made in [14].

Our main result is the following. We consider a topological group $X$ equipped with a proper length function $\ell: X \rightarrow \mathbb{R}_{+}$, a unitary representation $\mathrm{U}: X \rightarrow \mathscr{U}(\mathcal{H})$, and a net $\left\{x_{j}\right\}_{j \in J}$ in $X$ with $x_{j} \rightarrow \infty$ (see Section 2 for precise definitions). Also, we assume there exists an auxiliary self-adjoint operator $A$ in $\mathcal{H}$ such that the commutators $\left[A, U\left(x_{j}\right)\right]$ exist and are bounded in some precise sense, and such that the strong limit

$$
\mathrm{D}:=\mathrm{s}-\lim _{j} \frac{1}{\ell\left(x_{j}\right)}\left[A, \mathrm{u}\left(x_{j}\right)\right] \mathrm{u}\left(x_{j}\right)^{-1}
$$

exists. Then, under these assumptions we show that the unitary representation $\mathrm{U}$ is strongly mixing in $\operatorname{ker}(\mathrm{D})^{\perp}$ along the net $\left\{x_{j}\right\}_{j \in J}$ (Theorem 2.3). As a corollary, we obtain criteria for strong mixing in the cases of unitary representations of compactly generated locally compact Hausdorff groups (Corollary 2.5) and the Euclidean group $\mathbb{R}^{\mathrm{d}}$ (Corollary 2.7). These results generalise the commutator criteria of [14] for the strong mixing of discrete and continuous flows, as well as the strong limit (1.3) generalises the strong limits (1.1) and (1.2) (see Remarks 2.6] and 2.8). To conclude, we present in Example 2.9 an application which was not possible to cover with the results of [14]: a short alternative proof (not using convolutions) of the strong mixing property of the left regular representation of $\sigma$-compact locally compact Hausdorff groups. 
We refer the reader to $4,6,9,10,11,16$ for references on strong mixing properties of unitary representations of groups.

\section{Commutator criteria for strong mixing}

We start with a short review of basic facts on commutators of operators and regularity classes associated with them. We refer to [1, Chap. 5-6] for more details.

Let $\mathcal{H}$ be an arbitrary Hilbert space with scalar product $\langle\cdot, \cdot\rangle$ antilinear in the first argument, denote by $\mathscr{B}(\mathcal{H})$ the set of bounded linear operators on $\mathcal{H}$, and write $\|\cdot\|$ both for the norm on $\mathcal{H}$ and the norm on $\mathscr{B}(\mathcal{H})$. Let $A$ be a self-adjoint operator in $\mathcal{H}$ with domain $\mathcal{D}(A)$, and take $S \in \mathscr{B}(\mathcal{H})$. For any $k \in \mathbb{N}$, we say that $S$ belongs to $C^{k}(A)$, with notation $S \in C^{k}(A)$, if the map

$$
\mathbb{R} \ni \mathrm{t} \mapsto \mathrm{e}^{-i \mathrm{tA}} \mathrm{S} \mathrm{e}^{i \mathrm{tA}} \in \mathscr{B}(\mathcal{H})
$$

is strongly of class $C^{k}$. In the case $k=1$, one has $S \in C^{1}(A)$ if and only if the quadratic form

$$
\mathcal{D}(\mathrm{A}) \ni \varphi \mapsto\langle\varphi, \mathrm{iSA} \varphi\rangle-\langle\mathrm{A} \varphi, \mathrm{i} S \varphi\rangle \in \mathbb{C}
$$

is continuous for the topology induced by $\mathcal{H}$ on $\mathcal{D}(A)$. We denote by $[i S, A]$ the bounded operator associated with the continuous extension of this form, or equivalently the strong derivative of the map (2.1) at $t=0$. Moreover, if we set $A_{\varepsilon}:=(i \varepsilon)^{-1}\left(e^{i \varepsilon A}-1\right)$ for $\varepsilon \in \mathbb{R} \backslash\{0\}$, we have (see [1, Lemma 6.2.3(a)]):

$$
\underset{\varepsilon \backslash 0}{S-\lim _{\mathcal{1}}}\left[i S, A_{\varepsilon}\right]=[i S, A] .
$$

Now, if $\mathrm{H}$ is a self-adjoint operator in $\mathcal{H}$ with domain $\mathcal{D}(\mathrm{H})$ and spectrum $\sigma(\mathrm{H})$, we say that $H$ is of class $C^{k}(A)$ if $(H-z)^{-1} \in C^{k}(A)$ for some $z \in \mathbb{C} \backslash \sigma(H)$. In particular, $H$ is of class $C^{1}(A)$ if and only if the quadratic form

$$
\mathcal{D}(\mathrm{A}) \ni \varphi \mapsto\left\langle\varphi,(\mathrm{H}-z)^{-1} \mathrm{~A} \varphi\right\rangle-\left\langle\mathrm{A} \varphi,(\mathrm{H}-z)^{-1} \varphi\right\rangle \in \mathbb{C}
$$

extends continuously to a bounded form with corresponding operator denoted by $\left[(\mathrm{H}-z)^{-1}, \mathrm{~A}\right] \in$ $\mathscr{B}(\mathcal{H})$. In such a case, the set $\mathcal{D}(\mathrm{H}) \cap \mathcal{D}(A)$ is a core for $\mathrm{H}$ and the quadratic form

$$
\mathcal{D}(\mathrm{H}) \cap \mathcal{D}(\mathrm{A}) \ni \varphi \mapsto\langle\mathrm{H} \varphi, A \varphi\rangle-\langle A \varphi, \mathrm{H} \varphi\rangle \in \mathbb{C}
$$

is continuous in the topology of $\mathcal{D}(\mathrm{H})$ (see [1, Thm. 6.2.10(b)]). This form then extends uniquely to a continuous quadratic form on $\mathcal{D}(\mathrm{H})$ which can be identified with a continuous operator $[\mathrm{H}, \mathrm{A}]$ from $\mathcal{D}(\mathrm{H})$ to the adjoint space $\mathcal{D}(\mathrm{H})^{*}$. In addition, the following relation holds in $\mathscr{B}(\mathcal{H}$ ) (see [1, Thm. 6.2.10(b)]):

$$
\left[(H-z)^{-1}, A\right]=-(H-z)^{-1}[H, A](H-z)^{-1} .
$$


With this, we can now present our first result, which is at the root of the new commutator criterion for strong mixing. For it, we recall that a net $\left\{x_{j}\right\}_{j \in J}$ in a topological space $X$ diverges to infinity, with notation $x_{j} \rightarrow \infty$, if $\left\{x_{j}\right\}_{j \in J}$ has no limit point in $X$. This implies that for each compact set $K \subset X$, there exists $j_{K} \in J$ such that $x_{j} \notin K$ for $j \geq j_{K}$. In particular, $X$ is not compact. We also fix the notations $\mathscr{U}(\mathcal{H})$ for the set of unitary operators on $\mathcal{H}$ and $\mathbb{R}_{+}:=[0, \infty)$.

Proposition 2.1. Let $\left\{\mathrm{U}_{\mathfrak{j}}\right\}_{\mathfrak{j} \in \mathrm{J}}$ be a net in $\mathscr{U}(\mathcal{H})$, let $\left\{\ell_{j}\right\}_{j \in \mathrm{J}} \subset \mathbb{R}_{+}$satisfy $\ell_{\mathfrak{j}} \rightarrow \infty$, assume there exists a self-adjoint operator $\mathrm{A}$ in $\mathcal{H}$ such that $\mathrm{U}_{\mathrm{j}} \in \mathrm{C}^{1}(\mathrm{~A})$ for each $\mathrm{j} \in \mathrm{J}$, and suppose that the strong limit

$$
\mathrm{D}:=\mathrm{s}-\lim _{j} \frac{1}{\ell_{j}}\left[\mathrm{~A}, \mathrm{U}_{\mathrm{j}}\right] \mathrm{U}_{\mathrm{j}}^{-1}
$$

exists. Then, $\lim _{\mathfrak{j}}\left\langle\varphi, \mathrm{U}_{\mathrm{j}} \psi\right\rangle=0$ for all $\varphi \in \operatorname{ker}(\mathrm{D})^{\perp}$ and $\psi \in \mathcal{H}$.

Before the proof, we note that for $j \in J$ large enough (so that $\ell_{j} \neq 0$ ) the operators $\frac{1}{\ell_{j}}\left[A, U_{j}\right] U_{j}^{-1}$ are well-defined, bounded and self-adjoint. Therefore, their strong limit D is also bounded and self-adjoint.

Proof. Let $\varphi=\mathrm{D} \widetilde{\varphi} \in \mathrm{D} \mathcal{D}(A)$ and $\psi \in \mathcal{D}(A)$, take $j \in J$ large enough, and set

$$
D_{j}:=\frac{1}{\ell_{j}}\left[A, U_{j}\right] U_{j}^{-1} \text {. }
$$

Since $U_{j}$ and $u_{j}^{-1}$ belong to $C^{1}(A)$ (see [1, Prop. 5.1.6(a)]), both $u_{j} \psi$ and $u_{j}^{-1} \widetilde{\varphi}$ belong to $\mathcal{D}(A)$. Thus,

$$
\begin{aligned}
& \left|\left\langle\varphi, \mathrm{u}_{j} \psi\right\rangle\right| \\
& =\left|\left\langle\left(\mathrm{D}-\mathrm{D}_{j}\right) \widetilde{\varphi}, \mathrm{u}_{j} \psi\right\rangle+\left\langle\mathrm{D}_{j} \widetilde{\varphi}, \mathrm{U}_{j} \psi\right\rangle\right| \\
& \leq\left\|\left(\mathrm{D}-\mathrm{D}_{j}\right) \widetilde{\varphi}\right\|\|\psi\|+\frac{1}{\ell_{j}}\left|\left\langle\left[A, \mathrm{u}_{j}\right] \mathrm{U}_{j}^{-1} \widetilde{\varphi}, \mathrm{u}_{j} \psi\right\rangle\right| \\
& \leq\left\|\left(\mathrm{D}-\mathrm{D}_{j}\right) \widetilde{\varphi}\right\|\|\psi\|+\frac{1}{\ell_{j}}\left|\left\langle A \widetilde{\varphi}, \mathrm{U}_{j} \psi\right\rangle\right|+\frac{1}{\ell_{j}}\left|\left\langle\mathrm{U}_{j} A u_{j}^{-1} \widetilde{\varphi}, \mathrm{U}_{j} \psi\right\rangle\right| \\
& \leq\left\|\left(\mathrm{D}-\mathrm{D}_{j}\right) \widetilde{\varphi}\right\|\|\psi\|+\frac{1}{\ell_{j}}\|A \widetilde{\varphi}\|\|\psi\|+\frac{1}{\ell_{j}}\|\widetilde{\varphi}\|\|A \psi\| .
\end{aligned}
$$

Since $D=s-\lim _{j} D_{j}$ and $\ell_{j} \rightarrow \infty$, we infer that $\lim _{j}\left\langle\varphi, \mathrm{U}_{j} \psi\right\rangle=0$, and thus the claim follows by the density of $\mathrm{D} \mathcal{D}(\mathrm{A})$ in $\overline{\mathrm{DH}}=\operatorname{ker}(\mathrm{D})^{\perp}$ and the density of $\mathcal{D}(\mathrm{A})$ in $\mathcal{H}$.

In the sequel, we assume that the unitary operators $U_{j}$ are given by a unitary representation of a topological group $X$. We also assume that the scalars $\ell_{j}$ are given by a proper length function on $X$, that is, a function $\ell: X \rightarrow \mathbb{R}_{+}$satisfying the following properties ( $e$ denotes the identity of $X)$ :

(L1) $\ell(e)=0$, 
(L2) $\ell\left(x^{-1}\right)=\ell(x)$ for all $x \in X$,

(L3) $\ell(x y) \leq \ell(x)+\ell(y)$ for all $x, y \in X$,

(L4) if $\mathrm{K} \subset \mathbb{R}_{+}$is compact, then $\ell^{-1}(\mathrm{~K}) \subset \mathrm{X}$ is relatively compact.

Remark 2.2 (Topological groups with a proper left-invariant pseudo-metric). Let $\mathrm{X}$ be a Hausdorff topological group equipped with a proper left-invariant pseudo-metric $\mathrm{d}: \mathrm{X} \times \mathrm{X} \rightarrow \mathbb{R}_{+}$(see [7, Def. 2.A.5 8 2.A.7]). Then, simple calculations show that the associated length function $\ell: X \rightarrow \mathbb{R}_{+}$given by $\ell(x):=\mathrm{d}(e, x)$ satisfies the properties (L1)-(L4) above. Examples of groups admitting a proper left-invariant pseudo-metric are $\sigma$-compact locally compact Hausdorff groups 7 , Prop. 4.A.2], as for instance compactly generated locally compact Hausdorff groups with the word metric [7, Prop. 4.B.4(2)].

The next theorem provides a general commutator criterion for the strong mixing property of a unitary representation of a topological group. Before stating it, we recall that if a topological group $X$ is equipped with a proper length function $\ell$, and if $\left\{x_{j}\right\}_{j \in J}$ is a net in $X$ with $x_{j} \rightarrow \infty$, then $\ell\left(x_{j}\right) \rightarrow \infty$ (this can be shown by absurd using the property (L4) above).

Theorem 2.3 (Topological groups). Let $\mathrm{X}$ be a topological group equipped with a proper length function $\ell$, let $\mathrm{U}: \mathrm{X} \rightarrow \mathscr{U}(\mathcal{H})$ be a unitary representation of $\mathrm{X}$, let $\left\{x_{\mathfrak{j}}\right\}_{j \in \mathrm{J}}$ be a net in $\mathrm{X}$ with $\mathrm{x}_{\mathrm{j}} \rightarrow \infty$, assume there exists a self-adjoint operator $\mathrm{A}$ in $\mathcal{H}$ such that $\mathrm{U}\left(\mathrm{x}_{\mathrm{j}}\right) \in \mathrm{C}^{1}(\mathrm{~A})$ for each $\mathrm{j} \in \mathrm{J}$, and suppose that the strong limit

$$
\mathrm{D}:=\mathrm{s}-\lim _{\mathrm{j}} \frac{1}{\ell\left(\mathrm{x}_{\mathrm{j}}\right)}\left[\mathrm{A}, \mathrm{u}\left(\mathrm{x}_{\mathrm{j}}\right)\right] \mathrm{u}\left(\mathrm{x}_{\mathrm{j}}\right)^{-1}
$$

exists. Then,

(a) $\lim _{\mathrm{j}}\left\langle\varphi, \mathrm{U}\left(\mathrm{x}_{\mathrm{j}}\right) \psi\right\rangle=0$ for all $\varphi \in \operatorname{ker}(\mathrm{D})^{\perp}$ and $\psi \in \mathcal{H}$,

(b) U has no nontrivial finite-dimensional unitary subrepresentation in $\operatorname{ker}(\mathrm{D})^{\perp}$.

Proof. The claim (a) follows from Proposition 2.1 and the fact that $\ell\left(x_{j}\right) \rightarrow \infty$. The claim (b) follows from (a) and the fact that matrix coefficients of finite-dimensional unitary representations of a group do not vanish at infinity (see for instance [3, Rem. 2.15(iii)]).

Remark 2.4. (i) The result of Theorem 2.3(a) amounts to the strong mixing property of the unitary representation $\mathrm{U}$ in $\operatorname{ker}(\mathrm{D})^{\perp}$ along the net $\left\{\mathrm{x}_{\mathrm{j}}\right\}_{\mathrm{j} \in \mathrm{J}}$, as mentioned in the introduction. If the strong limit (2.4) exists for all nets $\left\{x_{j}\right\}_{j \in J}$ with $x_{j} \rightarrow \infty$, then Theorem [2.3(a) implies the usual strong mixing property of the unitary representation $\mathrm{U}$ in $\operatorname{ker}(\mathrm{D})^{\perp}$.

(ii) One can easily see that Theorem 2.3 remains true if the scalars $\ell\left(x_{j}\right)$ in (2.4) are replaced by $(f \circ \ell)\left(x_{\mathfrak{j}}\right)$, with $\mathrm{f}: \mathbb{R}_{+} \rightarrow \mathbb{R}_{+}$any proper function. For simplicity, we decided to present only the case $\mathrm{f}=\mathrm{id}_{\mathbb{R}_{+}}$, but we note this additional freedom might be useful in applications. 
Theorem 2.3 and Remark 2.2 imply the following result in the particular case of a compactly generated locally compact group $\mathrm{X}$ :

Corollary 2.5 (Compactly generated locally compact groups). Let X be a compactly generated locally compact Hausdorff group with generating set $\mathrm{Y}$ and word length function $\ell$, let $\mathrm{U}: \mathrm{X} \rightarrow \mathscr{U}(\mathcal{H})$ be a unitary representation of $\mathrm{X}$, let $\left\{\mathrm{x}_{\mathbf{j}}\right\}_{\mathfrak{j} \in \mathrm{J}}$ be a net in $\mathrm{X}$ with $\mathrm{x}_{\mathfrak{j}} \rightarrow \infty$, assume there exists a selfadjoint operator $\mathrm{A}$ in $\mathcal{H}$ such that $\mathrm{U}(\mathrm{y}) \in \mathrm{C}^{1}(\mathrm{~A})$ for each $\mathrm{y} \in \mathrm{Y}$, and suppose that the strong limit

$$
\mathrm{D}:=\mathrm{s}-\lim _{j} \frac{1}{\ell\left(x_{j}\right)}\left[A, \mathrm{u}\left(\mathrm{x}_{\mathrm{j}}\right)\right] \mathrm{u}\left(\mathrm{x}_{\mathrm{j}}\right)^{-1}
$$

exists. Then,

(a) $\lim _{\mathrm{j}}\left\langle\varphi, \mathrm{U}\left(\mathrm{x}_{\mathrm{j}}\right) \psi\right\rangle=0$ for all $\varphi \in \operatorname{ker}(\mathrm{D})^{\perp}$ and $\psi \in \mathcal{H}$,

(b) $\mathrm{U}$ has no nontrivial finite-dimensional unitary subrepresentation in $\operatorname{ker}(\mathrm{D})^{\perp}$.

Proof. In order to apply Theorem 2.3, we first note from Remark 2.2 that the word length function $\ell$ is a proper length function. Second, we note that $X=\bigcup_{n \geq 1}\left(Y \cup Y^{-1}\right)^{n}$. Therefore, for each $x \in X$ there exist $n \geq 1, y_{1}, \ldots, y_{n} \in Y$ and $m_{1}, \ldots, m_{n} \in\{ \pm 1\}$ such that $x=y_{1}^{m_{1}} \cdots y_{n}^{m_{n}}$. Thus,

$$
\mathrm{U}(\mathrm{x})=\mathrm{U}\left(\mathrm{y}_{1}^{\mathrm{m}_{1}} \cdots \mathrm{y}_{\mathrm{n}}^{\mathrm{m}_{\mathrm{n}}}\right)=\mathrm{U}\left(\mathrm{y}_{1}\right)^{\mathrm{m}_{1}} \cdots \mathrm{U}\left(\mathrm{y}_{\mathrm{n}}\right)^{\mathrm{m}_{\mathrm{n}}},
$$

and it follows from the inclusions $\mathrm{U}\left(\mathrm{y}_{1}\right), \ldots, \mathrm{U}\left(\mathrm{y}_{\mathrm{n}}\right) \in \mathrm{C}^{1}(A)$ and standard results on commutator methods [1, Prop. 5.1.5 \& 5.1.6(a)] that $\mathrm{U}(\mathrm{x}) \in \mathrm{C}^{1}(\mathrm{~A})$. Thus, we have $\mathrm{U}\left(\mathrm{x}_{\mathrm{j}}\right) \in \mathrm{C}^{1}(\mathrm{~A})$ for each $j \in J$, and the commutators $\left[A, U\left(x_{j}\right)\right]$ appearing in $(2.5)$ make sense. So, we can apply Theorem 2.3 to conclude.

Remark 2.6. Corollary [2.5 is a generalisation of [14. Thm. 3.1] to the case of unitary representations of compactly generated locally compact Hausdorff groups. Indeed, if we let $\mathrm{X}$ be the additive group $\mathbb{Z}$ with generating element 1 , take the trivial net $\left\{x_{j}=j\right\}_{j \in \mathbb{N}^{*}}=\left\{\mathrm{N} \mid \mathrm{N} \in \mathbb{N}^{*}\right\}$, and set $\mathrm{U}:=\mathrm{U}(1)$ in Corollary 2.5, then the strong limit (2.5) reduces to

$$
\mathrm{D}=\mathrm{s}_{\mathrm{N} \rightarrow \infty} \frac{1}{\mathrm{~N}}\left[\mathrm{~A}, \mathrm{U}^{\mathrm{N}}\right] \mathrm{U}^{-\mathrm{N}}=\mathrm{s}_{\mathrm{N} \rightarrow \infty} \frac{1}{\mathrm{~N}} \sum_{\mathrm{n}=0}^{\mathrm{N}-1} \mathrm{U}^{\mathrm{n}}\left([A, \mathrm{U}] \mathrm{U}^{-1}\right) \mathrm{U}^{-\mathrm{n}},
$$

which is the strong limit appearing in [14, Thm. 3.1]. In Corollary [2.5 we also removed the unnecessary invariance assumption $\eta(D) \mathcal{D}(A) \subset \mathcal{D}(A)$ for each $\eta \in \mathrm{C}_{\mathrm{c}}^{\infty}(\mathbb{R} \backslash\{0\})$. So, the strong mixing properties for skew products and Furstenberg-type transformations established in [14, Sec. 3] and [5. Sec. 3] can be obtained more directly using Corollary 2.5.

In the next corollary we consider the case of a strongly continuous unitary representation $\mathrm{U}: \mathbb{R}^{\mathrm{d}} \rightarrow \mathscr{U}(\mathcal{H})$ of the Euclidean group $\mathbb{R}^{\mathrm{d}}, \mathrm{d} \geq 1$. In such a case Stone's theorem implies the 
existence of a family of mutually commuting self-adjoint operators $H_{1}, \ldots, H_{d}$ such that $\mathrm{U}(\mathrm{x})=$ $\mathrm{e}^{-i \sum_{k=1}^{\mathrm{d}} x_{k} H_{k}}$ for each $x=\left(x_{1}, \ldots, x_{d}\right) \in \mathbb{R}^{\mathrm{d}}$. Therefore, we give a criterion for strong mixing in terms of the operators $H_{1}, \ldots, H_{d}$. We use the shorthand notations

$$
H:=\left(H_{1}, \ldots, H_{d}\right), \quad \Pi(H):=\left(H_{1}+i\right)^{-1} \cdots\left(H_{d}+i\right)^{-1} \quad \text { and } \quad x \cdot H:=\sum_{k=1}^{d} x_{k} H_{k} .
$$

Corollary 2.7 (Euclidean group $\left.\mathbb{R}^{\mathrm{d}}\right)$. Let $\mathbb{R}^{\mathrm{d}}$, $\mathrm{d} \geq 1$, be the Euclidean group with Euclidean length function $\ell$, let $\mathrm{U}: \mathbb{R}^{\mathrm{d}} \rightarrow \mathscr{U}(\mathcal{H})$ be a strongly continuous unitary representation of $\mathbb{R}^{\mathrm{d}}$, let $\left\{x_{j}\right\}_{j \in J}$ be a net in $\mathbb{R}^{\mathrm{d}}$ with $\mathrm{x}_{\mathbf{j}} \rightarrow \infty$, assume there exists a self-adjoint operator $\mathrm{A}$ in $\mathcal{H}$ such that $\left(\mathrm{H}_{\mathrm{k}}-\mathfrak{i}\right)^{-1} \in \mathrm{C}^{1}(\mathrm{~A})$ for each $\mathrm{k} \in\{1, \ldots, \mathrm{d}\}$, and suppose that the strong limit

$$
D:=s-\lim _{j} \frac{1}{\ell\left(x_{j}\right)} \int_{0}^{1} d s e^{-i s\left(x_{j} \cdot H\right)} \Pi(H)\left[i\left(x_{j} \cdot H\right), A\right] \Pi(H)^{*} e^{i s\left(x_{j} \cdot H\right)}
$$

exists. Then,

(a) $\lim _{\mathfrak{j}}\left\langle\varphi, \mathrm{U}\left(\mathrm{x}_{\mathrm{j}}\right) \psi\right\rangle=0$ for all $\varphi \in \operatorname{ker}(\mathrm{D})^{\perp}$ and $\psi \in \mathcal{H}$,

(b) U has no nontrivial finite-dimensional unitary subrepresentation in $\operatorname{ker}(\mathrm{D})^{\perp}$.

Proof. The proof consists in applying Theorem 2.3 with $A$ replaced by a new operator $\widetilde{A}$ that we now define.

The inclusions $\left(\mathrm{H}_{1}-\mathrm{i}\right)^{-1}, \ldots,\left(\mathrm{H}_{\mathrm{d}}-\mathfrak{i}\right)^{-1} \in \mathrm{C}^{1}(\mathrm{~A})$ and the standard result on commutator methods [1, Prop. 5.1.5] imply that $\Pi(H)^{*} \in C^{1}(A)$. So, we have $\Pi(H)^{*} \mathcal{D}(A) \subset \mathcal{D}(A)$, and the operator

$$
\widetilde{A} \varphi:=\Pi(\mathrm{H}) \mathrm{A} \Pi(\mathrm{H})^{*} \varphi, \quad \varphi \in \mathcal{D}(\mathrm{A}),
$$

is essentially self-adjoint (see [1, Lemma 7.2.15]). Take $\varphi \in \mathcal{D}(A)$ and $j_{0} \in J$ such that $\ell\left(x_{j}\right)>0$ for all $\mathfrak{j} \geq j_{0}$, and define for $\varepsilon \in \mathbb{R} \backslash\{0\}$ the operator $A_{\varepsilon}:=(i \varepsilon)^{-1}\left(e^{i \varepsilon A}-1\right)$. Then, we have

$$
\begin{aligned}
& \left\langle\widetilde{A} \varphi, \mathrm{U}\left(\mathrm{x}_{\mathrm{j}}\right) \varphi\right\rangle-\left\langle\varphi, \mathrm{U}\left(\mathrm{x}_{\mathrm{j}}\right) \widetilde{A} \varphi\right\rangle \\
& =\lim _{\varepsilon \searrow 0}\left(\left\langle\varphi, \Pi(H) A_{\varepsilon} \Pi(H)^{*} e^{-i\left(x_{j} \cdot H\right)} \varphi\right\rangle-\left\langle\varphi, \mathrm{e}^{-i\left(x_{j} \cdot H\right)} \Pi(H) A_{\varepsilon} \Pi(H)^{*} \varphi\right\rangle\right) \\
& =\lim _{\varepsilon \searrow 0} \int_{0}^{\ell\left(x_{j}\right)} \mathrm{dq} \frac{\mathrm{d}}{\mathrm{dq}}\left\langle\varphi, \mathrm{e}^{i\left(\mathrm{q}-\ell\left(x_{j}\right)\right)\left(x_{j} \cdot H\right) / \ell\left(x_{j}\right)} \Pi(H) A_{\varepsilon} \Pi(H)^{*} \mathrm{e}^{-i q\left(x_{j} \cdot H\right) / \ell\left(x_{j}\right)} \varphi\right\rangle \\
& =\frac{1}{\ell\left(x_{j}\right)} \lim _{\varepsilon \searrow 0} \int_{0}^{\ell\left(x_{j}\right)} \mathrm{d} q\left\langle\varphi, \mathrm{e}^{i\left(q-\ell\left(x_{j}\right)\right)\left(x_{j} \cdot H\right) / \ell\left(x_{j}\right)} \Pi(H)\left[i\left(x_{j} \cdot H\right), A_{\varepsilon}\right] \Pi(H)^{*} e^{-i q\left(x_{j} \cdot H\right) / \ell\left(x_{j}\right)} \varphi\right\rangle .
\end{aligned}
$$

But, $\left(\mathrm{H}_{1}-i\right)^{-1}, \ldots,\left(\mathrm{H}_{\mathrm{d}}-\mathrm{i}\right)^{-1} \in \mathrm{C}^{1}(\mathrm{~A})$. Therefore, (2.2) and (2.3) imply that

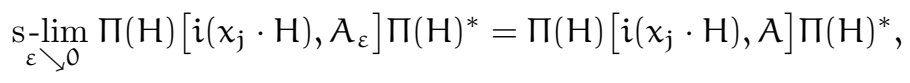


and we can exchange the limit and the integral in (2.7) to obtain

$$
\begin{aligned}
& \left\langle\widetilde{A} \varphi, \mathrm{u}\left(\mathrm{x}_{\mathrm{j}}\right) \varphi\right\rangle-\left\langle\varphi, \mathrm{u}\left(\mathrm{x}_{\mathrm{j}}\right) \widetilde{\mathrm{A}} \varphi\right\rangle \\
& =\frac{1}{\ell\left(x_{j}\right)} \int_{0}^{\ell\left(x_{j}\right)} d q\left\langle\varphi, e^{i\left(q-\ell\left(x_{j}\right)\right)\left(x_{j} \cdot H\right) / \ell\left(x_{j}\right)} \Pi(H)\left[i\left(x_{j} \cdot H\right), A\right] \Pi(H)^{*} e^{-i q\left(x_{j} \cdot H\right) / \ell\left(x_{j}\right)} \varphi\right\rangle \\
& =\frac{1}{\ell\left(x_{j}\right)} \int_{0}^{\ell\left(x_{j}\right)} \mathrm{dr}\left\langle\varphi, \mathrm{e}^{-i r\left(x_{j} \cdot H\right) / \ell\left(x_{j}\right)} \Pi(H)\left[i\left(x_{j} \cdot H\right), A\right] \Pi(H)^{*} e^{i\left(r-\ell\left(x_{j}\right)\right)\left(x_{j} \cdot H\right) / \ell\left(x_{j}\right)} \varphi\right\rangle \\
& =\int_{0}^{1} \mathrm{~d} s\left\langle\varphi, \mathrm{e}^{-i s\left(x_{j} \cdot H\right)} \Pi(H)\left[i\left(x_{j} \cdot H\right), A\right] \Pi(H)^{*} e^{i s\left(x_{j} \cdot H\right)} U\left(x_{j}\right) \varphi\right\rangle \\
& =\left\langle\varphi, \ell\left(x_{j}\right) D_{j} U\left(x_{j}\right) \varphi\right\rangle
\end{aligned}
$$

with

$$
D_{j}:=\frac{1}{\ell\left(x_{j}\right)} \int_{0}^{1} d s e^{-i s\left(x_{j} \cdot H\right)} \Pi(H)\left[i\left(x_{j} \cdot H\right), A\right] \Pi(H)^{*} e^{i s\left(x_{j} \cdot H\right)} .
$$

Since $\mathcal{D}(A)$ is a core for $\widetilde{A}$, this implies that $U\left(x_{j}\right) \in C^{1}(\widetilde{A})$ with $\left[\widetilde{A}, U\left(x_{j}\right)\right]=\ell\left(x_{j}\right) D_{j} U\left(x_{j}\right)$. Therefore, we have

$$
D_{j}=\frac{1}{\ell\left(x_{j}\right)}\left[\widetilde{A}, u\left(x_{j}\right)\right] u\left(x_{j}\right)^{-1},
$$

and all the assumptions of Theorem 2.3 are satisfied with $A$ replaced by $\widetilde{A}$.

Remark 2.8. Corollary 2.7 is a generalisation of [14, Thm. 4.1] to the case of strongly continuous unitary representations of $\mathbb{R}^{\mathrm{d}}$ for an arbitrary $\mathrm{d} \geq 1$. Indeed, if we set $\mathrm{d}=1$, write $\mathrm{H}$ for $\mathrm{H}_{1}$, and take the trivial net $\left\{x_{j}=j\right\}_{j \in(0, \infty)}=\{t \mid t>0\}$ in Corollary 2.7, then the strong limit (2.6) reduces to

$$
\begin{aligned}
D & =s_{t \rightarrow \infty} \frac{\lim }{t} \frac{1}{t} \int_{0}^{1} d s e^{-i s(t \cdot H)}(H+i)^{-1}[i t H, A](H-i)^{-1} e^{i s(t \cdot H)} \\
& =s_{t \rightarrow \infty}-\lim _{t \rightarrow \infty} \frac{1}{t} \int_{0}^{t} d s e^{-i s H}(H+i)^{-1}[i H, A](H-i)^{-1} e^{i s H},
\end{aligned}
$$

which is (up to a sign) the strong limit appearing in 14, Thm. 4.1]. In Corollary 2.7, we also removed the unnecessary invariance assumption $\eta(D) \mathcal{D}(A) \subset \mathcal{D}(A)$ for each $\eta \in \mathrm{C}_{\mathrm{c}}^{\infty}(\mathbb{R} \backslash\{0\})$. So, the strong mixing properties for adjacency operators, time changes of horocycle flows, etc., established in [14, Sec. 4] can be obtained more directly using Corollary 2.7.

To conclude, we add to the list of examples presented in [14] an application which was not possible to cover with the results of [14]. It is a short alternative proof, not using convolutions, of the strong mixing property of the left regular representation of $\sigma$-compact locally compact Hausdorff groups (see for instance [2, Sec. C.4] for the proof using convolutions): 
Example 2.9 (Left regular representation). Let $\mathrm{X}$ be a $\sigma$-compact locally compact Hausdorff group with left Haar measure $\mu$ and proper length function $\ell$ (see Remark 2.2). Let $\mathscr{D} \subset \mathcal{H}$ be the set of functions $\mathrm{X} \rightarrow \mathbb{C}$ with compact support, and let $\mathrm{U}: \mathrm{X} \rightarrow \mathscr{U}(\mathcal{H})$ be the left regular representation of $\mathrm{X}$ on $\mathcal{H}:=\mathrm{L}^{2}(\mathrm{X}, \mu)$ given by

$$
\mathrm{U}(\mathrm{x}) \varphi:=\varphi\left(\mathrm{x}^{-1} \cdot\right), \quad x \in \mathrm{X}, \varphi \in \mathcal{H} .
$$

Let finally $\mathrm{A}$ be the maximal multiplication operator in $\mathcal{H}$ given by

$$
\mathrm{A} \varphi:=\ell \varphi \equiv \ell(\cdot) \varphi, \quad \varphi \in \mathcal{D}(A):=\{\varphi \in \mathcal{H} \mid\|\ell \varphi\|<\infty\} .
$$

For $\varphi \in \mathscr{D}$ and $\mathrm{x} \in \mathrm{X}$, one has

$$
\operatorname{AU}(x) \varphi-\mathrm{U}(\mathrm{x}) \mathrm{A} \varphi=\left(\ell(\cdot)-\ell\left(\mathrm{x}^{-1} \cdot\right)\right) \mathrm{U}(\mathrm{x}) \varphi .
$$

Furthermore, the properties (L2)-(L3) of a length function imply that

$$
\left|\left(\ell(\cdot)-\ell\left(x^{-1} \cdot\right)\right)\right| \leq \ell(x) .
$$

Therefore, since $\mathscr{D}$ is dense in $\mathcal{D}(\mathrm{A})$, it follows that $\mathrm{U}(\mathrm{x}) \in \mathrm{C}^{1}(\mathrm{~A})$ with

$$
[A, U(x)] U(x)^{-1}=\ell(\cdot)-\ell\left(x^{-1} \cdot\right) .
$$

Now, we take $\left\{\mathrm{x}_{\mathbf{j}}\right\}_{\mathbf{j} \in \mathrm{J}}$ a net in $\mathrm{X}$ with $\mathrm{x}_{\mathbf{j}} \rightarrow \infty$, and show that

$$
\mathrm{D}:=\mathrm{s}-\lim _{\mathbf{j}} \frac{1}{\ell\left(x_{\mathfrak{j}}\right)}\left[A, \mathrm{U}\left(\mathrm{x}_{\mathfrak{j}}\right)\right] \mathrm{U}\left(\mathrm{x}_{\mathfrak{j}}\right)^{-1}=-1 .
$$

For this, we first note that for $\varphi \in \mathcal{H}$ we have

$$
\left(\frac{1}{\ell\left(x_{j}\right)}\left[A, U\left(x_{j}\right)\right] U\left(x_{j}\right)^{-1}+1\right) \varphi=\frac{\ell(\cdot)-\ell\left(x_{j}^{-1} \cdot\right)+\ell\left(x_{j}\right)}{\ell\left(x_{j}\right)} \varphi .
$$

Next, we note that (2.8) implies that

$$
\left|\frac{\ell(\cdot)-\ell\left(x_{j}^{-1} \cdot\right)+\ell\left(x_{j}\right)}{\ell\left(x_{j}\right)} \varphi\right|^{2} \leq 4|\varphi|^{2} \in L^{1}(X, \mu),
$$

and that the properties (L2)-(L3) imply that

$$
\lim _{j}\left|\frac{\ell(\cdot)-\ell\left(x_{j}^{-1} \cdot\right)+\ell\left(x_{j}\right)}{\ell\left(x_{j}\right)} \varphi\right|^{2} \leq \lim _{j}\left|\frac{2 \ell(\cdot)}{\ell\left(x_{j}\right)} \varphi\right|^{2}=0 \quad \mu \text {-almost everywhere. }
$$

Therefore, we can apply Lebesgue dominated convergence theorem to get the equality

$$
s-\lim _{j}\left(\frac{1}{\ell\left(x_{j}\right)}\left[A, U\left(x_{j}\right)\right] U\left(x_{j}\right)^{-1}+1\right) \varphi=0,
$$

which proves (2.9). So, Theorem 2.3 applies with $\mathrm{D}=-1$, and thus $\lim _{\mathrm{j}}\left\langle\varphi, \mathrm{U}\left(\mathrm{x}_{\mathrm{j}}\right) \psi\right\rangle=0$ for all $\varphi, \psi \in \mathcal{H}$. 


\section{References}

[1] W. O. Amrein, A. Boutet de Monvel, and V. Georgescu. Co-groups, commutator methods and spectral theory of $\mathrm{N}$-body Hamiltonians, volume 135 of Progress in Mathematics. Birkhäuser Verlag, Basel, 1996.

[2] B. Bekka, P. de la Harpe, and A. Valette. Kazhdan's property (T), volume 11 of New Mathematical Monographs. Cambridge University Press, Cambridge, 2008.

[3] M. B. Bekka and M. Mayer. Ergodic theory and topological dynamics of group actions on homogeneous spaces, volume 269 of London Mathematical Society Lecture Note Series. Cambridge University Press, Cambridge, 2000.

[4] V. Bergelson and J. Rosenblatt. Mixing actions of groups. Illinois J. Math. 32(1): 65-80, 1988.

[5] P. A. Cecchi and R. Tiedra de Aldecoa. Furstenberg transformations on cartesian products of infinite-dimensional tori. Potential Analysis 44(1): 43-51, 2016.

[6] R. Cluckers, Y. de Cornulier, N. Louvet, R. Tessera, and A. Valette. The Howe-Moore property for real and p-adic groups. Math. Scand. 109(2): 201-224, 2011.

[7] Y. de Cornulier and P. de la Harpe. Metric geometry of locally compact groups, volume 25 of EMS Tracts in Mathematics. European Mathematical Society (EMS), Zürich, 2016.

[8] C. Fernández, S. Richard, and R. Tiedra de Aldecoa. Commutator methods for unitary operators. J. Spectr. Theory 3(3): 271-292, 2013.

[9] R. E. Howe and C. C. Moore. Asymptotic properties of unitary representations. J. Funct. Anal. 32(1): 72-96, 1979.

[10] A. Lubotzky and S. Mozes. Asymptotic properties of unitary representations of tree automorphisms. In Harmonic analysis and discrete potential theory (Frascati, 1991), pages 289-298. Plenum, New York, 1992.

[11] K. Schmidt. Asymptotic properties of unitary representations and mixing. Proc. London Math. Soc. (3) 48(3): 445-460, 1984.

[12] R. Tiedra de Aldecoa. The absolute continuous spectrum of skew products of compact lie groups. Israel J. Math. 208(1): 323-350, 2015.

[13] R. Tiedra de Aldecoa. Spectral analysis of time changes of horocycle flows. J. Mod. Dyn. 6(2): 275-285, 2012. 
[14] R. Tiedra de Aldecoa. Commutator criteria for strong mixing. Ergodic Theory and Dynam. Systems 37(1): 308-323, 2017.

[15] R. Tiedra de Aldecoa. Commutator methods for the spectral analysis of uniquely ergodic dynamical systems. Ergodic Theory Dynam. Systems 35(3): 944-967, 2015.

[16] R. J. Zimmer. Ergodic theory and semisimple groups, volume 81 of Monographs in Mathematics. Birkhäuser Verlag, Basel, 1984. 